\title{
NEW VIEWS ON DIAGNOSTIC METHODS FOR THE FORMATION OF RETENTION OF THE UPPER PERMANENT CANINES (LITERATURE REVIEW)
}

\author{
Bakaev Zh.N., \\ Olimov S.Sh., \\ Bukhara State Medical Institute named after Abu Ali ibn Sino
}

\begin{abstract}
The article presents new views on methods for diagnosing the formation of retention of the upper permanent canines. The author presented a wide review of the literature of both foreign and domestic scientists, detailed the various diagnostic methods for this pathology. Various methods for assessing X-ray studies and modern approaches to diagnosing retention of the upper permanent canines, which are used in practice by an orthodontist, are also described. Keywords. Dental anomalies, orthopantomography, canine retention, cone-beam computed tomography.

Retained tooth (from Latin - holding, restraining) - a tooth that will not be able to erupt properly, as it is completely or partially covered by bone or thick gum tissue.
\end{abstract}

\section{Introduction}

Worldwide data of Russian and foreign authors, the referral of patients with this pathology for surgical and orthodontic help is different. In the structure of treatment of patients with dental anomalies (SAD) in Russia, according to studies, from 4.0 to $18.0 \%$ of patients with retention of the upper permanent canines. Abroad, the prevalence of retention of the upper permanent canines, according to the latest data, is significantly lower, which ranges from 0.8 to $3.0 \%$ [63].

Resorption of the roots by a row of standing teeth, the formation of odontogenic tumors, anomalous projection of adjacent teeth, a violation of the seizure and biting off of food - this is a whole series of morphological breakdowns in itself, which are behind This, in order, leads to a number of aesthetic claims that are made by patients with retention of the upper permanent canines, including a violation of the smile line, divergence of the center of the dentition, anomaly of the position of the teeth, face asymmetry $[10 ; 12 ; 38 ; 65]$.

The endocrine factors related to the functions of the endocrine glands, which are of great importance for the functioning of the child, significantly influencing the formation of his dentition and the development of the oral mucosa, were examined. They can influence the onset of dental anomalies during both prenatal and postnatal development of the child. Hypothyroidism, endemic goiter, idiopathic form of hypoparathyroidism, pseudohypoparathyroidism, cerebral pituitary dwarfism, Fröhlich's disease, rickets, according to many domestic and foreign authors, can lead to an abnormal position and violation of the timing of eruption $[35 ; 50 ; 56]$.

In addition to the above general factors, the development of the child's dentition is influenced by the unfavorable state of the environment: lack of fluorine in fresh water, insufficient ultraviolet irradiation, excessive radioactive background [3; eight; 49].

Retention of permanent canines can arise as a result of caries, its complications and the associated removal of individual deciduous teeth, more often in children there is a loss of temporary molars, which leads to displacement of adjacent teeth, eruption anomalies or mesial eruption of the first permanent molars. The result of the mesial displacement of the first permanent molars is the shortening of the dentition in the area of the support zone and the lack of space for the eruption of the canine [23;26].

Chronic apical destructive processes of milk teeth determine position anomalies and violations of the timing of the eruption of permanent teeth as a result of displacement of their primordia by granulation tissue, which is confirmed by a number of studies $[32 ; 35 ; 69]$.

Since the bone crypt of the primordium of a permanent tooth is connected by a guide canal with a temporary tooth, the preservation of the temporary predecessor is an important point in the eruption of a permanent tooth [18]. This intraosseous canal contains a fibrous cord with remnants of the epithelium of the dental plate and unites the crypt with the cortical plate and sometimes with the wall of the alveoli of the milk tooth. Features of the structure of the intraosseous canal have a significant impact on the eruption of the tooth, however, the diameter of this stroke varies depending on the size of the tooth. The crown diameter of a permanent canine is much larger than that of its predecessor. Thus, the creation of sufficient space for the eruption of permanent canines depends on bone and root resorption of the primary tooth $[65 ; 66]$. Delayed prolapse of milk canine is a consequence, not a cause, of permanent canine dystopia [59].

\section{Main part}

The roots of the lateral incisors are characterized by another guide for the eruption of permanent upper canines. To resist the pressure from the crowns of eruption canines, the roots of the lateral incisors, in a child of eight or nine 
years, should be sufficiently formed [15, 68]. As a result, the final eruption of the canines should lead to straightening the position of the incisors and the formation of adequate interdental contacts. The canine eruption may deviate either vestibularly or palatally relative to the dental arch, in the absence of contact between the canine crown and the root of the lateral incisor. Adentia or microdentia of the lateral incisors leads to the absence of a guide for the eruption of canines $[20 ; 21 ; 4 ; 64]$.

Super-complete teeth located in the direction of permanent complete teeth often cause their retention. This phenomenon is called hyperdontia. More than $80 \%$ of supercomplete teeth are found on the upper jaw and $90 \%$ of them are located in its anterior part $[62 ; 63]$.

Pressure on the rudiments of the corresponding permanent teeth from cysts or tumor foci can also cause retention [56]. Odontoma is the most common odontogenic neoplasm, consisting of abnormal or incorrectly differentiated odontogenic tissues [59; 60]. Clinically, an odontoma is almost identical to a super-complete tooth and can also counteract the development and eruption of permanent canines.

Odontogenic cysts are most often associated with a violation of the eruption of permanent teeth. Upper canines are affected more often than other teeth. Sometimes the cause of a cyst is pulpitis of the baby tooth. After excision of the neoplasm, the tooth can erupt independently if there is sufficient space in the dental arch [35; 45].

Shortening and narrowing of the upper dentition, which is also associated with the upper micrognathia, leads to a lack of space for all complete teeth, which may be one of the reasons for canine retention[7;23;24;27;36].

It is not always possible to divide the factors that lead to retention of permanent canines of the upper jaw into General and local, endogenous and exogenous, so this division, according to many authors, is conditional [ 28; 29; 32].

In Volgograd residents, etiological factors leading to tooth retention were systematized into groups. When canine retention occurs, the author identified the following as the main reasons: atypical laying of the rudiments of canines or adjacent teeth, supercomplete teeth, odontomas and cysts in the course of eruption, premature mineralization of the tip of the eruption canine [25].

Another compatriot on the basis of the results of their research suggested that clinico-morphological classification of the anomalies of the timing of eruption of permanent teeth, which takes into account the following etiological factors: a lack of space in the tooth row, the anomaly position reportaugust tooth, anomaly size and shape neporotovskiy teeth, supernumerary teeth, congenital disorders of the maxillofacial region [9].

It is proved that it is possible to establish the retention of teeth only on the basis of x-ray examination of the alveolar parts of the jaws $[7 ; 20 ; 49 ; 71]$. Intraoral $\mathrm{x}$-ray techniques, orthopantomography, telerentgenography, multispiral computed tomography, cone-beam computed tomography are currently used for the diagnosis of retenated and dystopian teeth $[31 ; 43 ; 46,57]$.

Intraoral radiography is performed on dental $\mathrm{x}$-ray diagnostic devices. It includes the following types of research: contact, occlusal and interproximal radiography. Intraoral radiography is of limited use in the diagnosis of retenated and dystopian teeth, since it does not provide a complete picture of the state of the dental system. However, it can be used to detect the presence of the tooth germ and its condition, determine the stage of formation and development of the tooth root, assess the state of pereapical tissues, detect super-complete teeth, determine the prospects for tooth eruption, and identify a pathological focus of a limited nature $[11 ; 16 ; 17,51]$.

Since 1987, radiovisiography has been developed in dentistry. Computer processing of information increases the diagnostic information content of the study by manipulating the contrast, brightness, clarity, size by eliminating technical errors, highlighting areas of interest. The advantages of radiovisiography are also a significant reduction in the radiation load, the ability to archive information [30].

The method of conducting orthopantomography (optg) was proposed in 1939 by Blackman, then mathematically justified and prepared for wide practical application by Finnish specialists Soila and Paatero (1956) [41]. When performing optg, the doctor is able to evaluate both jaws, teeth, temporomandibular joints, and paranasal sinuses, which allows determining the degree of mineralization of crowns and roots of teeth, their formation, and the stage and type of resorption of the roots of temporary teeth, which is important for diagnosing possible pathology of the structures of hard tissues of teeth. According to the optg data, it is possible to detect the rudiments of non-eruption teeth, determine their position in the jaw and the prospects of eruption [61]. In addition, orthopantomography is performed to determine the inclination of erupted teeth and retented teeth in relation to neighboring ones [31].

To determine the position of the crowns of impacted canines of the upper jaw in the vestibular-oral direction in their research, suggested the use of the principles based on the laws of optics: 1) radiographic shadow of the impacted tooth, is located the Palatine, on DPRs will always be more symmetrical tooth; 2) radiographic shadow of the impacted tooth situated deep in the alveolar process, on the DPRs will be closer in size to the shadow of the symmetric tooth; 3 ) the x-ray shadow of a retented tooth located vestibularly on the orthopantomogram will always be smaller than the size of a symmetrical tooth; 4) the edge sharpness of a tooth more distant from the film plane will always be lower than the shadow of a tooth located closer to the film plane [31].

In 2009, a study was conducted at Manchester medical University, where, according to a questionnaire survey of dentists, 22 dental centers that have an x-ray Department analyzed the popularity of this technique in dental practice. It was found that $73.3 \%$ of the surveyed doctors consider orthopantomography to be the most informative method in comparison with intraoral $\mathrm{x}$-ray examinations of teeth and periapical tissues for dental therapists during endodontic treatment, for dental surgeons when planning surgery, as well as for orthodontists in the diagnosis and treatment of dental retention [54]. Thus, orthopantomography has long served as the main method of radiological examination of 
patients with retented teeth [17, 37]. The method of orthopantomography, despite its great informative value, has a number of disadvantages. The spatially selected image layer is a strictly vertical but U-shaped horizontal plane with a thickness of 1-2 cm. The largest percentage of distortions, overlays, and mismatches occur in the front section. The image of the frontal part of the jaws in the image may be overlaid with the shadow of the spine and the lumen from the uncompressed tongue. The magnification of objects in the image can be from 1: 1.2 to 1: 1.75, depending on the design of the device and the area of the image [41].

Telerentgenography was proposed by the Italian anthropologist Paccini in 1922. In orthodontics, it was first used in 1931 by Hofrath in Germany and Broadbent in the United States, and in 1934 it was proposed to perform cephalometric analysis using a telerentgenogram. The method is used in orthodontics to diagnose the consequences of improper development of the jaws (according to Andresen), incorrect position of the teeth, occlusion disorders, jaw shape, and joint pathology. Despite certain drawbacks, the method is part of a survey that can technically classify numerous variants of dysgnathia depending on skeletal and dentoalveolar ratios. In the orthodontic clinic teleroentgenogram produce both direct and lateral projections. To correctly assess the retention of teeth, it is impractical to use it without orthopantomography [48; 51].

Localization of the upper permanent canines can be established using standard x-ray methods, with image distortion, superimposition of three-dimensional structures, artifacts, projection errors, and sometimes poor image quality $[57 ; 58]$.

Computed tomography is currently the most informative method of radiation diagnostics in dentistry and maxillofacial surgery [5, 42]. Many Russian and foreign authors consider computed tomography as a priority method for studying patients with dystopia and dental retention $[6 ; 19 ; 52]$.

The first CT scanner was tested in 1974. Later, its creators, engineers Cormack and Housefield, received the Nobel prize for this invention. Despite the broadest diagnostic capabilities, until now, computed tomography, as a method of examining patients with dental anomalies, has been extremely rarely used in dentistry. This was due to the high radiation load from the study and the fact that in most cases the CT scan had an image quality that was insufficient for the needs of dentists [40].

Until recently, there were three types of CT scanners: spiral, sequential, and magnetic resonance [19; 41; 60]. One of the first comparative radiological studies was conducted at the medical University of Vienna in 1995. Orthopantomography and computed tomography were used to study 29 patients with 36 permanent retented teeth. 2 programs (normal and dental) were used in the analysis. The authors summarized the following: 1) both computed tomography programs are visually more informative than orthopantomography; 2) the dental computed tomography program is more effective than the usual program in the diagnosis of retention, since it allows more accurate diagnosis of root resorption of neighboring teeth [68].

At the Institute of radiology of Pavia, research was carried out on spiral computed tomography to determine the localization of retented maxillary canines and further treatment planning. For this purpose, 19 patients were examined with 29 retenated permanent canines located differently in the thickness of the alveolar process (Palatine and vestibular). Patients were sequentially examined using orthopantomography, telerentgenography in lateral projection, and spiral computed tomography. As a result of the research, the authors found that it is impossible to detect resorption of the root of the adjacent incisor on the orthopantomogram, especially on its Palatine and buccal surfaces. Computed tomography easily diagnosed the space between the retenated canine and the adjacent incisor in 26 clinical cases, and resorption of the root of the adjacent incisor in 8 clinical cases [44].

The authors concluded that computed tomography facilitates the diagnosis of retented canines, especially when it is tilted to the top of the alveolar ridge of the upper jaw, reduces the time of radiological examination, and reduces the risk of possible movement of the patient's head [61].

At the Hokkaido medical University, a radiological study was conducted on the issue of three-dimensional assessment of retented incisors, canines, premolars, and molars of the upper jaw at the stage of surgical intervention planning. The authors compared images of the root shapes of retented teeth from intraoral images, orthopantomograms, and CT scans in 27 patients. With statistical confidence ( $\mathrm{P}$ " 0.01), the authors proved the difference in the reliability of the obtained $\mathrm{x}$-ray information and concluded that only computed tomography can make an accurate diagnosis of retention, with a clear definition of the shape of the root of the retented tooth in three-dimensional space [62].

The use of spiral computed tomography in patients with retention of the upper permanent canines was also studied by Russian authors. In 2007, based on a comparison of x-ray examination methods, the author revealed that spiral computed tomography is the most optimal additional method for assessing the location of the retented canine in three planes, determining its location relative to neighboring teeth, evaluating the morphology of the surface of the canine roots and adjacent teeth in the upper jaw, and detecting resorption of the roots of neighboring teeth on the retention side. Based on the observations made, the researcher developed a Protocol for x-ray examination of patients with retention of canines in the upper jaw, which allows planning orthodontic treatment based on optg data, occlusal radiographs, spiral computed tomography (CT), and TRG in the lateral projection. The advantages of the presented Protocol are that it systematizes the diagnostic process when planning treatment of patients with retention of the upper permanent canines and clearly defines the indications for SCT. The disadvantages include the need for a large number of x-ray studies, including the SCT method, in addition, this method has a high radiation load. The Protocol is used only for the purpose of diagnostic examination of patients with retention of the upper permanent canines and cannot be applied if their eruption is delayed. 
Other domestic author on the basis of the data of spiral computed tomograms, proposed a mathematical model created by means of a personal electronic computer (PC), allowing to finish editing entered data and implement computers using the estimated amount of free space to predict the treatment outcome of patients with impacted upper permanent canines [10].

Multispiral computed tomography with 3D reconstruction was applied in 2013 on the basis of the Moscow state medical and dental University (MSMSU) to develop a computer program for calculating the trajectory of retraction of a retented tooth. This method allows the doctor to simulate the position in a real situation, change the trajectory of movement taking into account the applied force vector, and visually predict the results of treatment [45, 53].

The most innovative direction of radiation diagnostics of the XXI century in dentistry was the creation and active implementation of cone-beam computed tomography. In the literature, there are various names for this research method, but according to the version of the European Academy of Dental Radiology (European Academy of DentoMaxilloFacial Radiology) and the American International Institute of Cone Beam tomography (International Cone Beam Institute), the method is called cone beam computed tomography (CBCT) [40; 67].

CBCT provides high-quality $\mathrm{x}$-ray images of the maxillofacial system and the maxillofacial region in three mutually perpendicular planes. The fundamental difference between specialized dental tomographs and sequential and spiral CT scans is that, first, in this case, a single planar sensor is used for scanning, and, secondly, that the generated beam is collimated in the form of a cone. During shooting, the emitter operates continuously, and information is read from the sensor several times a second. Then the information is processed in a computer and a virtual three-dimensional model of the scanned area is restored [52; 62].

Having all the advantages of multispiral computed tomography, cone-beam computed tomography allows performing a similar study with a lower radiation load, which is extremely important in children who make up the largest group of patients with retention of the upper permanent canines [22; 39]. Lower radiation load during CBCT is achieved due to the fact that the value of the current and anode voltage (voltage) in installations is many times less $70-90 \mathrm{kV}$ and 3-8 mA, for MSCT these values are 120-140 kV and $100 \mathrm{~mA}$, respectively. Thus, the radiation load from a single study varies from 0.04 to $0.08 \mathrm{mSv}$, depending on the type of tomograph [65]. According to SanPiN 2.6.1.80299 , for practically healthy individuals, the annual effective dose during preventive medical radiological procedures should not exceed $1 \mathrm{mSv}$. Based on these data, the method of cone-beam computed tomography can be attributed to low-dose studies [1]. In contrast to conventional radiography, cone-beam computed tomography is the most informative, since it allows you to obtain images of any anatomical formations in three planes, select a layer of the object of interest and a number of organ complexes located from 1 to $10 \mathrm{~mm}$ thick [53]. Alqerban (2011) determined that CBCT is significantly better than panoramic radiography for determining the level of root resorption of lateral incisors in the categories of small and heavy resorption [60]. The largest comparative study was a prospective study in which data from radiation research methods were analyzed by seven independent doctors. At the same time, patients underwent traditional (intraoral and panoramic) and high-tech (cone-beam computed tomography) x-ray methods. Experts independently developed a treatment plan for each patient (using data from either traditional research methods or high-tech ones). Discussing the results of x-ray examination, calculating statistically the accuracy of the diagnosis and the correctness of the chosen treatment, it was concluded that for the diagnosis of dental anomalies and the choice of treatment tactics, it is more appropriate to use cone-beam computed tomography [55].

However, according to a number of authors, this method of diagnosis has not been widely used due to the lack of a clear systematic methodology that allows for comprehensive diagnostic studies $[47 ; 66]$.

A group of Russian researchers proposed an informative and systematic method for the diagnosis of retented teeth using cone-beam computed tomography (Sirona "GALILEOS“, Morita"3DX"). The method included: 1) determining the location of the retented teeth in the anterior and lateral areas of the jaws, as well as the angle of inclination of the longitudinal axes of the retented teeth to the coordinate axes; 2) determining the distance from the retented tooth to the compact plate of the jaw; 3) determination of the bone density in the area of retented teeth and comparison with the bone density in the area of the same teeth on the opposite side. The method offers an algorithm for studying CBCT data, but does not provide a total assessment of the severity of tooth retention [46].

Another researcher described the relationship between the distance of the retenirovynny canines of the upper jaw from the occlusal plane and the probability of their removal. This pattern is called the "rule of vertical thirds" and can be used for predicting treatment $[51 ; 52]$.

A group of foreign researchers in 2009 proposed a method for estimation of CBCT, taking into account the number of parameters of the position of impacted canines: angle of inclination, vertical position relative to the occlusal plane, anterior-posterior position of the root apex and the degree of overlap of adjacent cutter. Based on the evaluation of these parameters, the author determines the forecast of eruption of the upper permanent canines [58].

Foreign researchers in 2015 publications point to the fact that there is no single agreement in orthodontic practice, according to which criteria for determining the possibility of orthodontic treatment of retented canines would be allocated. As a result of a literature review of 237 articles devoted to the retention of upper permanent canines, the authors identified 10 parameters for determining the difficulty of treating retenated canines using CBCT data. So, according to the authors, the complexity of orthodontic treatment for patients with impacted upper permanent canines is influenced by such factors as: the age of the patient; the position of the impacted tooth in three planes; the presence of impacted tooth transposition with the lateral incisor or the first premolar, the presence of dilacerate the root of the impacted tooth, the presence of root resorption of the adjacent teeth. In conclusion, the authors point out that the most 
important parameters for assessing the difficulty of treating retented canines using CBCT data are the patient's age, the slope relative to the occlusal plane, dilaceration, the shadow of the retented canine on neighboring teeth, and the distance to the occlusal plane [46].

Based on the variety of etiological factors that lead to retention of the upper permanent canines, we can conclude that it is necessary to dynamically monitor and conduct regular preventive examinations for the eruption of these teeth in children, starting from the period of replacement bite [2].

\section{Conclusion}

Thus, summing up the literature review of 87 articles, various X-ray research methods can be used to diagnose the retention of upper permanent canines. The most modern currently available is CBCT, which combines the acquisition of a large volume of diagnostic data with low radiation exposure to the patient. The literature describes various methods for evaluating x-ray studies to diagnose and plan treatment of retention of the upper permanent canines, but there are no works related to x-ray evaluation of the dental system of children during the period of replacement bite in order to prevent the formation of retention of canines. Still, we have no right to judge and insist on using this or that technique for x-ray studies, because much depends on the economic development of a particular region. Also, not a little depends on the orthodontist, namely in the ability to read information received by a particular resource.

\section{References}

1. "Hygienic requirements for the device and operation of x-ray rooms, apparatuses and x-ray examinations". San. Pin. 2.6.1.802-99.

2. Asimova sh. sh., Abruev U. R, Rasulov M. M. Tish-zhag tizimi anomalyalaring Bukhoro Shahar Maktab yeshdagi Bolalar orasida tarkalishi. // Doctor Akhborotnomasi. 2020. - No. 1. - Pp. 23-28 bet.

3. Anikienko A. A. Clinical manifestations and etiology of eruption disorders of permanent teeth / A. A. Anikienko // Orthodontics. - 2000. - № 1-2. - P. 57-60.

4. Arzhantsev A. P. Methods of x-ray research and x-ray diagnostics in dentistry / A. P. Arzhantsev. - Moscow, 2015. - 260C.

5. Balin V. N. Experience of using computed tomography in the diagnosis of diseases of the maxillary region / V. N. Balin // Dentistry. - 1994. - No. 1. - Pp. 30-32.

6. Bimbas E. S. Differential diagnosis and treatment of varieties of mesial bite: dis. ... candidate of medical Sciences / E. S. Bimbas. Perm, 1989, Pp. 143-145.

7. Bragin E. A. Application of computed tomography in the diagnosis and treatment of retenated teeth / E. A. Bragin // Orthodontics. — 2004. — № 2 (26). — Pp. 43-45. 118

8. Bragin A. E. Assessment of gnatological indicators of patients with anomalies of the terms of eruption of permanent teeth: dis. ... candidate of medical Sciences / A. E. Bragin. - Stavropol, 2011. - 21 p.

9. Vakushina E. A. Efficiency of modern methods of diagnostics and treatment in complex rehabilitation of patients with anomalies of the position and timing of eruption of permanent teeth: dis. ... Cand. honey. Sciences / E. A. Makushina. - Volgograd, 2007.

10. Vakushina E. A. Prevalence of retention of permanent teeth / E. A. Vakushina // New in the theory and practice of dentistry: Collection of scientific papers. - Sevastopol, 2007. - P. 244-248.

11. Williams S. Conceptual orthodontics II. Growth and orthopedics / S. Williams. - Lviv: Galdent, 2006. - 200

p.

12. gafforov S. A., Bakaev Zh. n. Features of the relationship between diseases of the oral mucosa and somatic pathology //Herald of the TMA. - Tashkent, 2019. - No. 3. - P. 20-24.

13. Gafforov S. A., Bakayev Zh. Ois BglII chilli avati of kasalliklari of pathologic aspekter VA asoslari prevention // Stomatologiya. - Tashkent, 2019. - №4 (77). - P. 75-80.

14. Gasimova Z. Impacted teeth: diagnosis and integrated treatment / Z. V. Gasimova // Orthodontics. - 2002. No. 2. - P. 2-7.

15. Glukhova Yu. M. Analysis of the causes and clinical manifestations of combined pathology (retention of teeth and malocclusion) in adolescents and adults according to the cone beam computed tomography / Y. M. Glukhov, N. S. Shpak, And V. P. Sinyakov // Institute of dentistry. - 2016. - No. 70. - Pp. 72-73.

16. Glukhova Yu. m. Clinical justification of the diagnostic and therapeutic complex for patients with dental anomalies complicated by periodontal diseases / Yu. M. Glukhova, A. I. Kiryutina / / Institute of dentistry. - 2012. - No. 1. - Pp. 62-64.

17. Doroshenko S. I. Clinic and differential diagnostics of various forms of retention in children / S. I. Doroshenko, E. A. Kulginsky / / Suchasna orthodontia. — 2010. — № 01 (19). — P. 3-13. 120

18. Elizarova V. M. Dentistry of children: textbook. In 3 hours / V. M. Elizarova. - Part 1. - M.: GEOTARMedia, 2016. - $480 \mathrm{p}$.

19. Ishmuratova A. F. Relevance of timely diagnostics of dental retention in the conditions of mass dental care / A. F. Ishmuratova, G. V. Stepanov // Stomatology of children's age and prevention. - 2011. - No. 3. - Pp. 61-63.

20. Ishmuratova A. F. Prevalence of hypodentia in the Samara region / A. F. Ishmuratova // In the collection: Achievements of orthodontics, orthopedic and General dentistry in the specialty and its teaching. - M., 2012. - Pp. 104- 
107.

21. Ishmuratova A. F. Comparative assessment of the degree of complexity of the diagnosed dental anomaly in children with primary partial adentia in the framework of a comprehensive program of detection, dental treatment and rehabilitation depending on the age of patients and the method of organization [Electronic resource] / A. F. Ishmuratova, A.V. Anokhina, T. I. Sadykova // Modern problems of science and education. - 2012. - No. 6. - P. 200. Mode of access: https://elibrary.ru/contents.asp?issueid=1381400.

22. Yohan K. M. Radiation load during X-ray imaging of teeth and maxillofacial region in pediatric dentistry / K. M. Yohan. - Moscow: dental-Expo, 2013. - P. 3-7.

23. Kalivrajiyan E. S. Analysis of treatment methods for narrowing and shortening of dentition in the temporary and initial stage of a removable bite / E. S. Kalivrajiyan, S. I. burlutskaya / / Journal of practical and theoretical biology and medicine. - 2003. - Vol. 2, No. 4. - P. 335-336.

24. Karakotov L. Sh. Features of diagnostics and complex treatment of patients with unilateral Vestibuloocclusion: dis. ... candidate of medical Sciences / L. sh. Karakotova. - Stavropol, 2010. - 143 p.

25. Komarova T. V. Methods of moving retented teeth with orthodontic devices with a dosed load: dis. ... candidate of medical Sciences / T. V. Komarova. - Volgograd, 2000. - 119 p.

26. Lazareva E. A. Study of the prevalence and prerequisites for the occurrence of canine retention / E. A. Lazareva // Orthodontics. - 2013. - No. 2. - P. 53.

27. Lazareva E. A. Features of peripheral blood flow in the area of retenated canines of the upper jaw / E. A. Lazareva / / Dental Forum. - 2014. - No. 4. - C. 66-68.

28. Lazareva E. A. Features of cephalometric parameters in patients with retention of upper jaw canines / E. A. Lazareva / / Actual problems of pediatric dentistry and prevention: Mat. III regional conference dedicated to the 25th anniversary of the Department of pediatric dentistry. - Khabarovsk, 2013. - P. 117-120. 123

29. Lazareva E. A. Comparative analysis of methods of early orthodontic treatment in order to create a place for permanent canines of the upper jaw in children in late replacement bite / E. A. Lazareva // Orthodontics. - 2014. - no. 3. - Pp. 77-79.

30. Radiation diagnostics in dentistry / A. Yu. Vasiliev, Yu. I. Vorobyov, N. S. Serova, etc. - Moscow: GEOTAR-Media, 2008. - 201-220 p.

31. Mazen SHUK. Clinical and radiological diagnostics and hardware-surgical treatment of canine retention: dis. ... candidate of medical Sciences / SHUK Mazen. - Tver, 2004.

32. the Microvascular tone of periodontal tissues and its reactive response to treatment using non-removable orthodontic techniques / E. A. Cardboard, N. V. snetkova, S. N. ermolyev, etc. / / Orthodontics. - 2013. - No. 2. - Pp. 45-46.

33. millet D. Solving problems in orthodontics and pediatric dentistry / D. millet, R. Welbury. - Medpressinform, 2009. - 200 p. 124

34. Nechaeva N. K. Cone-beam tomography in dental implantology / N. K. Nechaeva. - M.: GEOTAR-Media, 2016. $-96 \mathrm{p}$.

35. Olimov S. sh. the Value of metabolic parameters of saliva in the development of dental anomalies in children with metabolic syndrome. / / "Stomatologist kasalliklarni davolashda VA preventacidagi dolzarb muammolar" Ilmiy Amali konferentsiyasi. Buhoro. 2016. - 33bet.

36. Olimov, S. S., Gafarov S. A. Bollard Tish-a of tizimi nucelar VA somatic kasalliklar orasida boiling of Ilmi asoslari. (Adabelle share).// Journal. Stomatologiya. - $2019 \# 1.60-65$ bet.

37. Olimov, S. S., Saidov, A. A., S. A. Gaffarov, Hojimetov A. A. Maktab esday of bollard hepatobiliar of tizimning Tish-a noseley of pathogeneticaly aliate.// Doctor akhborotnomasi magazine. Samarkand. 2019 - \# 2. - 35-39 bet.

38. Olimov S. sh. Evaluation of the effectiveness of preventive measures in the orthodontic treatment of children with dental deformities./ / Ykuv-uslubiy kyllanma. SR CERs of tomonidan 13.11.08 tastier. Tashkent. 2019. - 21 b.

39. Application of cone-beam computed tomography in surgical dentistry, maxillofacial surgery and otorhinolaryngology for differential diagnosis of dental diseases / A. L. Dudarev, A. A. Zubareva, M. A. Chibisova, M. A. Shavgulidze // Dentist-practitioner. - 2012. - no. 4. - P. 20-25.

40. Rogatskin D. V. Radiodiagnostics of the maxillofacial region. Cone-beam computed tomography. Fundamentals of visualization / D. V. Rogatkin. - Lviv: Galdent, 2010. - 148 p.

41. Rogatskin D. V. Panoramic tomography of dental rows. Methodological recommendations / D. V. Rogatskin. - $\mathrm{SPb}$.: Man, 2010. - $48 \mathrm{p}$.

42. Serova N. S. Radiation diagnostics in dental implantology / N. S. Serova. - M.: GEOTAR-Media, 2015. - 220

p.

43. Stepanov G. V. Complex diagnostics and treatment of bite anomalies in dental alveolar shortening: dis. ... dRA med. Sciences / G. V. Stepanov. - Samara, 2011. - 43 p.

44. Stepanov G. V. Resorption of tooth roots in orthodontic treatment / V. G. Stepanov, A. F. Ishmuratova // Endodontics Today. - 2011. - No. 1. - P. 52-53.

45. Topolnitsky O. Z. Dentistry of children: textbook. In 3 hours / O. Z. Topolnitsky. - Part 2. - Moscow: GEOTAR-Media, 2016. - 320 p. 
46. Fadeev R. A. Method for assessing the position of retented teeth according to dental computed tomography / R. A. Fadeev, Yu. P. Shveleva, M. A. Chibisova // Institute of dentistry. - 2010. - No. 47. - P. 16-18.

47. Fleischer G. Propaedeutics of children's orthopedic dentistry / G. Fleischer. - LAP, 2014. - 68 p.

48. Friedrich Pasler A. X-ray Diagnostics in the practice of the dentist / Friedrich A. Pasler, Heiko Wissler. - M.: Medpress-inform, 2007. - P. 118-131.

49. Khamadeeva a.m. Prevention of dental diseases in ecologically unfavorable regions on the example of Chapaevsk / a.m. Khamadeeva, N. V. Nogina, A. F. Ishmuratova // Prevention of dental diseases and oral hygiene: Mat. III Russian scientific and practical conference. - Kazan, 2010. - P. 198-201. 